\title{
« Le soleil de la prospérité actuelle ne luit pas pour tout le monde ": les exclus de la société de consommation à Montréal,
} 1945-1975

\section{STÉPHANIE O’NEILL}

Volume 70, numéro 4, printemps 2017

URI : https://id.erudit.org/iderudit/1040574ar

DOI : https://doi.org/10.7202/1040574ar

Aller au sommaire du numéro

Éditeur(s)

Institut d'histoire de l’Amérique française

ISSN

0035-2357 (imprimé)

1492-1383 (numérique)

Découvrir la revue

Citer cette note

O’Neill, S. (2017). « Le soleil de la prospérité actuelle ne luit pas pour tout le monde » : les exclus de la société de consommation à Montréal, 1945-1975. Revue d'histoire de l'Amérique française, 70(4), 55-70.

https://doi.org/10.7202/1040574ar

\section{Résumé de l'article}

Cette note de recherche fait ressortir quelques indicateurs permettant de mieux connaître les ménages qui demeurent hors de la société de consommation en voie de consolidation à Montréal entre 1945 et 1975. En ayant recours aux données statistiques et à divers travaux, surtout contemporains de la période à l'étude, elle procède à une mise en lumière de quelques aspects de la géographie de la pauvreté de la ville et des municipalités adjacentes ainsi que des appartenances sociales des ménages exclus de l'abondance des Trente Glorieuses. Il en ressort qu'hier comme aujourd'hui, la pauvreté étend davantage son emprise sur certains quartiers de la ville ainsi que sur les Autochtones, les membres de certaines communautés culturelles, les familles nombreuses et les ménages dont la chef est une femme. 


\title{
Note de recherche
}

\author{
"Le soleil de la prospérité actuelle \\ ne luit pas pour tout le monde ${ }^{\mathrm{n}}$ : \\ les exclus de la société de \\ consommation à Montréal, \\ $1945^{-1975}$
}

\section{STÉPHANIE O’NEILL \\ Docteure en histoire}

\begin{abstract}
RÉsumÉ • Cette note de recherche fait ressortir quelques indicateurs permettant de mieux connaître les ménages qui demeurent hors de la société de consommation en voie de consolidation à Montréal entre 1945 et 1975. En ayant recours aux données statistiques et à divers travaux, surtout contemporains de la période à l'étude, elle procède à une mise en lumière de quelques aspects de la géographie de la pauvreté de la ville et des municipalités adjacentes ainsi que des appartenances sociales des ménages exclus de l'abondance des Trente Glorieuses. II en ressort qu'hier comme aujourd'hui, la pauvreté étend davantage son emprise sur certains quartiers de la ville ainsi que sur les Autochtones, les membres de certaines communautés culturelles, les familles nombreuses et les ménages dont la chef est une femme.
\end{abstract}

ABSTRACT - This research note highlights some of the main characteristics of households that remained outside of consumer society during a period of relative prosperity, between 1945 and 1975 in Montréal. Relying on statistical surveys and various studies, most of which were performed during the period under study, it describes aspects of the geography of poverty in the city and neighbouring municipalities, and examines the social makeup of households excluded from prosperity within a so-called affluent society. It shows that, much like today, some neighbourhoods and some groups — namely indigenous peoples, members

1. «Le soleil de la prospérité actuelle ne luit pas pour tout le monde», Le Travail (novembre 1964): 6.

2. Cette recherche a bénéficié du soutien financier du Conseil de recherche en sciences humaines, du Groupe d'histoire de Montréal ainsi que de la Faculté des études supérieures et postdoctorales et du département d'histoire de l'Université de Montréal. L'auteure tient aussi à remercier Denyse Baillargeon pour sa lecture attentive de versions préliminaires de cette note de recherche ainsi que les évaluateurs anonymes de la RHAF. 
of certain ethnic communities, large families, and households with female heads - were more likely to be in a state of economic hardship.

Trop nombreux sont nos concitoyens et concitoyennes, personnes âgées, veuves, malades, jeunes ménages, immigrants, salariés qui n’ont pas encore été touchés par la grâce d'une économie en expansion³.

Pendant la période de prospérité relative surnommée les Trente Glorieuses, les revenus des ménages augmentent, l'inflation demeure faible et l'emploi connaît des années de vache grasse, surtout par rapport à la décennie et demie de privations qui la précède ou aux années de stagflation qui la suivent. Les revenus progressent de façon impressionnante, les salaires horaires réels moyens faisant plus que doubler dans le secteur manufacturier entre 1945 et $1975^{4}$. Ainsi, de manière générale, «le pouvoir d'achat de la majorité des Québécois s'améliore " pendant l'après-guerre et dans les années 1960, ce qui se répercute inévitablement sur leur niveau de vie. Bon nombre de Montréalais peuvent désormais participer à la société de consommation qui se met alors en place. On assiste à un essor impressionnant dans la consommation domestique, notamment en ce qui a trait aux appareils électroménagers, aux automobiles et aux téléviseurs. Qui dit généralisation de la consommation de masse, dit également démocratisation du confort matériel et société plus égalitaire : en apparence, le ciel est donc au beau fixe.

Bien que la société québécoise se meuve dans une prospérité jusque-là inégalée, pour bien des baby boomers montréalais, alors enfants ou adolescents, et leurs parents, la réalité est tout autre. Dans les faits, des poches de pauvreté sont présentes tout au long de la période et tranchent avec l'image idyllique des banlieues d'après-guerre ${ }^{6}$, même si on assiste effectivement à

3. Émile Gosselin, La troisième solitude. Une étude de la pauvreté dans la région de Montréal par le Conseil du Travail de Montréal F.T.Q. - C.T.C. (Montréal, 1965), 78.

4. Jacques Rouillard et Jean-François Rouillard, «Salaires et productivité du travail au Canada depuis le $\mathrm{XX}^{\mathrm{e}}$ siècle : les travailleurs bénéficient-ils de la croissance économique?», Relations industrielles/Industrial Relations 70, 2 (2015), graphique 6, 364. Rouillard et Rouillard adoptent toutefois une périodisation différente de la nôtre.

5. Paul-André Linteau et al., Histoire du Québec contemporain, tome II : Le Québec depuis 1930 (Montréal, Boréal, 2007 [1989]), 320.

6. Plusieurs historiens ont en effet nuancé le portrait bien uniforme et un peu trop rose de banlieues soi-disant homogènes et exclusivement composées de la classe moyenne. Au Québec, Jean-Pierre Collin et Claire Poitras ont démontré que les municipalités faisant partie de l'entité désignée Rive-sud de Montréal ne sont pas indifférenciées, tandis que Paul-André Linteau a noté la diversité des banlieues d'après-guerre dans la région montréalaise. Plus encore, il remarque que des «secteurs situés en périphérie [de Montréal] prennent l'allure de véritables bidonvilles et attirent une population défavorisée à la 
un exode vers la périphérie de familles qui ont les moyens de devenir propriétaires ${ }^{7}$. Au Québec, même les tenants du «mythe» de la Grande Noirceur, pourtant prompts à souligner les tares de l'époque duplessiste, ne rendent pas compte de ces exclus de la prospérité, à Montréal comme ailleurs. Hormis quelques historiens, notamment Magda Fahrni qui a observé que, pour bien des familles montréalaises, la consommation de biens durables ou l'achat d'une propriété en banlieue reste inaccessible, du moins pendant les années qui suivent le second conflit mondial ${ }^{8}$, l'historiographie demeure peu loquace quant à leur existence.

Cela s'explique peut-être par le fait qu'en dehors des documents produits par les associations caritatives et les institutions gouvernementales, peu de sources laissent entrevoir la persistance de la pauvreté en milieu urbain pendant l'après-guerre bien qu'elle constitue le lot d'une frange considérable de la population. En s'appuyant sur des données statistiques et sur divers rapports gouvernementaux, il est néanmoins possible de faire ressortir quelques traits des ménages qui demeurent hors de la société de consommation en voie de consolidation à Montréal, ce que nous proposons de faire en mettant en lumière certains aspects de leur situation géographique et leurs appartenances sociales. Si leur nombre surprend par son importance, les lignes de faille qui ressortent de ce bref survol de la pauvreté urbaine sont moins étonnantes, bon nombre d'entre elles étant

recherche de logements à très bon marché. C’est notamment le cas de ville Jacques-Cartier, avec ses maisons recouvertes de papier goudron et ses infrastructures insuffisantes ", un phénomène dont Collin et Poitras soulignent aussi l'existence. Au Canada anglais, Veronica Strong-Boag a montré que même si une homogénéité ethnique, socioéconomique et, de plus en plus, visuelle caractérise les communautés suburbaines entre 1945 et 1960 , celles-ci sont toutefois potentiellement différentes les unes des autres, ce qui suggère que cet environnement reproduisait et confortait les clivages de la société canadienne d'après-guerre. Il faut ainsi garder en tête que les banlieues canadiennes d'après-guerre sont marquées par des différences régionales et n'abritent pas exclusivement des "[w]hite middle-class WASPs", même si ce groupe y est très présent. En ce qui concerne les États-Unis, l'historienne Lizabeth Cohen a illustré la persistance, voire l'exacerbation, des inégalités dans les banlieues de la consumer's republic qui se met en place après la Seconde Guerre mondiale. Jean-Pierre Collin et Claire Poitras, "La fabrication d'un espace suburbain. La rive-sud de Montréal», Recherches sociographiques, 43, 2 (2002): 286, 290 ; Paul-André Linteau, Histoire de Montréal depuis la Confédération (Montréal, Boréal, 1992), 494; Veronica Strong-Boag, "Home Dreams. Women and the Suburban Experiment in Canada, 1945-1960", Canadian Historical Review, 72, 4 (décembre 1991): 471-475, 483, 486-487, 492, 501-504; Lizabeth Cohen, A Consumers' Republic. The Politics of Mass Consumption in Postwar America (New York, Vintage Books, 2004). Voir également la description de Longueuil-Annexe (une partie de Ville Jacques-Cartier) dans les années 1940 de Pierre Vallières dans Nègres blancs d'Amérique (Montréal, éditions parti-pris, 1968), chapitre 2.

7. Entre 1951 et 1971 , la population de la région métropolitaine de recensement de Montréal double presque (elle passe de 1395400 à 2743208 habitants), tandis que moins de 200000 individus viennent grossir les rangs de la ville de Montréal dont la population passe de 1021520 à 1214352 pour la même période. P.-A. Linteau, Histoire de Montréal..., op. cit., 493.

8. Magda Fahrni, Household Politics. Montreal Families and Postwar Reconstruction (Toronto, University of Toronto Press, 2005): 15 . 
toujours d'actualité un demi-siècle plus tard. Hier comme aujourd'hui, elle étend davantage son emprise sur certains quartiers de la ville ainsi que sur les francophones, les Autochtones, les familles issues de certaines communautés culturelles et les ménages dont la chef est une femme'.

Ce portrait ne saurait être statique, mais les sources ne permettent pas vraiment de tirer des conclusions fermes sur les transformations de ce phénomène à Montréal pendant les Trente Glorieuses. Nous ne disposons en effet que d'une quantité restreinte de données sur la pauvreté urbaine pendant les années 1940 et 1950, possiblement parce qu'elle est si répandue qu'elle semble «normale». En fait, les "pauvres» ou la "pauvreté» font l'objet de beaucoup plus d'études à partir des années 1960, alors que le phénomène commence à être appréhendé en lien avec la société de consommation. Dès lors, plus de documents - c'est là un euphémisme - se penchent sur la population dont la consommation de masse n'améliore pas, ou pas assez, de l'avis des différents commentateurs ou intervenants, le niveau de vie, d'où la plus grande facilité pour nous d'en peindre le tableau.

Cela dit, esquisser la géographie de la pauvreté à Montréal constitue une entreprise plus ardue qu'il n'y paraît de prime abord. Celle-ci se heurte non seulement aux transformations des intérêts des instigateurs des collectes de données qui ne sont pas toujours préoccupés par les inégalités économiques, mais aussi au caractère historiquement et culturellement spécifique (du seuil) de la pauvreté tributaire de la définition fluctuante du nécessaire et du superflu. Même dans le cas où cette dernière ferait consensus, le revenu - familial ou du chef de famille - sur lequel s'appuie ce seuil n'est pas une mesure adéquate de la pauvreté. En effet, d'autres facteurs entrent en ligne de compte dans la situation économique d'un ménage qui dépend non seulement du salaire, mais aussi du nombre de bouches à nourrir, du travail domestique, de l'accès au crédit, de la propension et de la capacité des proches à offrir un soutien financier et de toutes les stratégies que les individus et les ménages peuvent mettre en œuvre. Plus encore, pour quiconque adopte les Trente Glorieuses comme périodisation, plusieurs seuils de pauvreté seraient nécessaires pour fournir une vue d'ensemble d'une époque où les salaires, les prix et les normes de consommation domestique varient considérablement. Ce

9. Nous ne pouvons insister sur leur situation ici, mais les ménages composés de personnes seules, de plus en plus nombreux pendant les Trente Glorieuses, sont particulièrement et distinctement touchés par la précarité économique, tout comme les aînés. 
phénomène n'est probablement pas sans lien avec l'absence de seuil de pauvreté officiel au Canada pendant cette période ${ }^{10}$.

Ces bémols n'atténuent toutefois pas l'utilité du recours aux statistiques : fournir quelques chiffres permet de prendre le pouls d'aspects de la pauvreté telle que définie par différents auteurs et organismes, mais surtout de sa persistance à Montréal pendant une ère de prospérité relative. La plupart du temps, les ressources dont les pauvres disposent et à partir desquelles ils sont jugés "pauvres» renvoient, dans les statistiques, au revenu, indicateur partiel, mais clair et tangible. C'est sur celui-ci que se basent des estimations officielles des années 1960 et 1970 selon lesquelles, au Canada et au Québec, c'est respectivement entre 20 et $28 \%$ des familles non agricoles dont le revenu est insuffisant ${ }^{11}$. En se servant des chiffres de Statistique Canada, le Comité spécial du Sénat sur la pauvreté indique pour sa part que $25 \%$ des Canadiens évoluent dans une famille sous le seuil de la pauvreté en $1969^{12}$. Si nous ne disposons pas de chiffres du genre pour les années d'immédiat après-guerre, il est légitime de penser que la situation va en s'améliorant, ou du moins, ne se détériore pas, compte tenu de la prospérité grandissante qui, malgré tout, caractérise l'ensemble de la période. Des politiques sociales qui sont ou entrent en vigueur pendant les Trente Glorieuses, comme les allocations familiales fédérales et des programmes universels «telles les pensions de vieillesse, l'assurancehospitalisation et l'assurance-maladie [auxquels] s'ajoutent des programmes de sécurité du revenu comme l'assurance-chômage et l'aide sociale $^{13}$ » contribuent en outre, peut-on présumer, à faire profiter un plus grand nombre de ménages de la prospérité ambiante. En effet, au Canada, la part des prestations sociales dans les revenus de l'ensemble des familles

10. David A. Croll, La pauvreté au Canada. Rapport du Comité spécial du Sénat sur la pauvreté (Ottawa, Imprimeur de la Reine, 1971): 220.

11. Economic Council of Canada, Fifth Annual Review. The Challenge of Growth and Change (Ottawa, Imprimeur de la Reine, 1968), 109; Commission d'enquête sur la santé et le bien-être social (Québec), La sécurité du revenu. Volume V, Tome 1: La situation actuelle/Les fondements d'une politique de sécurité du revenu - Rapport (Castonguay-Nepveu) de la Commission d'enquête sur la santé et le bien-être social (Québec, Éditeur officiel du Québec, 1971), 43. À titre indicatif, la Commission d'enquête sur la santé et le bien-être social (Québec), citant des données provenant d'études effectuées pour le compte d'organismes relevant du gouvernement fédéral, estime le "niveau d'insuffisance du revenu " pour une famille de 2 adultes et 2 enfants vivant au Canada à $3500 \$$ en 1961, à $4060 \$$ en 1967 et à $4771 \$$ en 1971. Pour une famille comptant 2 adultes et 5 enfants, ces chiffres s'élèvent respectivement à $5000 \$, 5800 \$$ et $6815 \$$ (p. 35-38).

12. D. A. Croll, La pauvreté au Canada..., op. cit., 11. Ce rapport est un peu moins précis quant à la source du revenu des familles, mais en offre un portrait plus global.

13. P. -A. Linteau, Histoire de Montréal..., op. cit., 512. 
et des personnes seules qui sont situées dans le quintile inférieur passe de $29 \%$ en 1951 à $45 \%$ en $1965^{14}$.

Selon La troisième solitude, une recherche commanditée par le Conseil du travail de Montréal et abondamment citée par la suite, la pauvreté - c'està-dire, pour une famille de 4 personnes, vivre avec un revenu annuel inférieur à $3000 \$$, ou pour une personne seule, inférieur à $1500 \$$ - est le lot de $20 \%$ des familles ${ }^{15}$ de la ville (et de $17 \%$ de la région métropolitaine) en $1961^{16}$. En outre, 50000 familles vivent «dans la privation», c'est-à-dire que, bénéficiant de moins de 4000 \$ par année, elles ne "peuvent tout au plus que joindre les deux bouts, sans pouvoir réaliser le moindre projet ${ }^{17}$ ", et sont vulnérables tant aux fluctuations de l'économie qu'aux aléas de l'existence. En incluant les personnes seules qui gagnent moins de $2000 \$$ par année, soit 453418 individus, c'est $38 \%$ des habitants de la ville qui connaissent soit la pauvreté ou la privation ${ }^{18}$. Dans l'ensemble de la zone métropolitaine de Montréal, ce serait "près de la moitié (48.6\%) [sic] de la population [qui pourrait] être classée comme économiquement faible ${ }^{19} »$. Au fil du temps, la situation semble s'améliorer. En effet, pour la période comprise entre 1967 et 1976, le Conseil économique du Canada (CEC) fournit des données un peu plus optimistes et, surtout, plus précises grâce, entre autres, à leur caractère longitudinal et à leur prise en compte de la taille des familles ${ }^{20}$. À partir d'un seuil de pauvreté propre à chaque métropole canadienne, le CEC indique ainsi que le pourcentage de la population pauvre à Montréal passe de 18 à $12 \%$ entre ces deux dates. Ces estimations concordent avec les données canadiennes et québécoises, étant donné que la pauvreté touche davantage les familles agricoles.

Selon toute vraisemblance, la pauvreté teinte donc le quotidien d'un nombre de plus en plus restreint de foyers montréalais, ce qui ne signifie pas qu'elle disparaisse. Dans une rétrospective sur les revenus des Canadiens de 1951 à 1981, Statistique Canada, en divisant les familles et les personnes seules en quintiles, démontre d'ailleurs qu'en 1951, 1961, 1971 et 1981, les écarts entre les revenus de tous les quintiles demeurent exactement les

14. Division du revenu et des dépenses des Consommateurs, Coup d'œil sur les revenus des Canadiens de 1951 à 1981 (Ottawa, Statistique Canada, 1984), 18.

15. Ces chiffres n'incluent pas les personnes seules.

16. E. Gosselin, La troisième solitude..., op. cit., 14, 25.

17. Ibid.

18. Ibid., 3 .

19. Ibid., 6.

20. Ludwig Auer et Kathryn McMullen, Discussion Paper no. 164. Changes in Poverty in Canadian Metropolitan Centres, 1967-1976 (Ottawa, Economic Council of Canada, 1980), 10-11. 
mêmes ou presque ${ }^{21}$. Dans le contexte où la pauvreté en vient de plus en plus à être comprise par les différents "experts» comme relative au sens de tributaire des standards de la société de consommation ${ }^{22}$, force est de constater qu'elle perdure tout au long de la période.

\section{LA GÉOGRAPHIE DE LA PAUVRETÉ MONTRÉALAISE}

Il ne fait donc aucun doute que des poches de pauvreté persistent tout au long des Trente Glorieuses. Si les milieux ruraux sont particulièrement touchés, les centres urbains ne sont pas épargnés, des quartiers montréalais étant aussi laissés pour compte de la prospérité ${ }^{23}$. Certes, maints ménages de la ville, grâce à l'augmentation de leurs revenus, peuvent migrer vers les banlieues, accéder à la propriété résidentielle et se procurer les moyens de transport allant de pair avec l'étalement urbain, mais c'est loin d'être le cas de tous. En fait, ou peut-être en conséquence, puisque bien des familles qui en ont les moyens prennent le chemin de la périphérie, le territoire montréalais est le théâtre d'une concentration de la pauvreté pendant la période à l'étude. Au début des années 1950, «les ménages aux revenus modestes [sont] relativement dispersés dans l'agglomération $^{24}$ » de Montréal comme le remarquent Anne-Marie Séguin, Paula Negron-Poblete et Philippe Apparicio. Le tableau 1 illustre cette dispersion, le revenu médian des chefs de famille salariés de la Cité de Montréal (2374\$) rejoignant presque celui de l'ensemble de la zone métropolitaine de Montréal qui tourne autour de $2420 \$$ en 1951 . Selon Séguin et al., à cette époque, les ménages à faibles revenus habitent ainsi non seulement les quartiers centraux, mais aussi «des zones plus périphériques [...] situées au nord, à l'ouest et à l'est de l'île, et dans la portion sud de Mercier-Ouest; sur la rive sud, à Jacques-Cartier (maintenant faisant partie de l'arrondissement du Vieux-Longueuil) et, à Laval, dans un secteur de Chomedey ${ }^{25}$ ».

21. Division du revenu et des dépenses des Consommateurs, Coup d'œil sur..., op. cit., 6.

22. À ce sujet, voir: Stéphanie O’Neill, «"Y aura-t-il toujours des pauvres ?” : les transformations des discours sur la pauvreté en période de prospérité ", Labour/Le Travail, 79 (printemps 2017): 1957-1984.

23. Paul-André Linteau et al., Histoire du Québec contemporain, Tome II : Le Québec depuis 1930 (Montréal, Boréal, 2007 [1989]), 207.

24. Anne-Marie Séguin et al., «Pauvreté et richesse dans la région montréalaise depuis l'après-guerre. Un paysage en mouvement », dans Dany Fougères, dir., Histoire de Montréal et de sa région, Tome II: De 1930 à nos jours (Québec, Les Presses de l’Université Laval, 2012), 1147.

25. Id. 
Tableau I

Évolution des revenus, île de Montréal, I 95 I - I 97 I, dollars courants

\begin{tabular}{|c|c|c|c|}
\hline & $\begin{array}{c}\text { I95 I } \\
\text { Revenu médian } \\
\text { du chef de } \\
\text { famille salarié (\$) }\end{array}$ & $\begin{array}{c}196 \mathbf{I}^{*} \\
\text { Revenu du chef } \\
\text { de famille salarié } \\
(\$)\end{array}$ & $\begin{array}{c}\text { I97 I } \\
\text { Revenu moyen } \\
\text { de l'emploi } \\
\text { du chef de famille (\$) }\end{array}$ \\
\hline $\begin{array}{l}\text { Zone métropolitaine } \\
\text { de Montréal }\end{array}$ & 2420 & 4407 & 8060 \\
\hline Cité de Montréal & 2374 & 3962 & 6796 \\
\hline Anjou & 2242 & 5223 & 8481 \\
\hline Côte-Saint-Luc & 3765 & 7199 & 12122 \\
\hline Hampstead & 5926 & 9721 & 19778 \\
\hline Lasalle & 2550 & 4426 & 7676 \\
\hline Montréal-Est & 2572 & 4254 & 6841 \\
\hline Montréal-Nord & 2290 & 4107 & 7148 \\
\hline Montréal-Ouest & 5083 & 8984 & 15343 \\
\hline Mont-Royal & 5747 & 10311 & 19438 \\
\hline Outremont & 2865 & 4781 & II 209 \\
\hline Pierrefonds & 2085 & 5730 & 10062 \\
\hline Saint-Laurent & 2892 & 5398 & 9077 \\
\hline Saint-Léonard & 2426 & 4551 & 7658 \\
\hline Saint-Michel & 2378 & 3744 & - \\
\hline Saint-Pierre & 2406 & 3862 & 6692 \\
\hline Verdun & 2572 & 4080 & 6967 \\
\hline Westmount & 3445 & 7715 & 19034 \\
\hline $\begin{array}{l}\text { Notre-Dame- } \\
\text { de-Liesse }\end{array}$ & I 875 & - & - \\
\hline $\begin{array}{l}\text { Municipalité de } \\
\text { Saint-Laurent }\end{array}$ & 2413 & - & - \\
\hline
\end{tabular}

* Il n'est pas spécifié s'il s'agit du revenu médian ou moyen.

Sources: Bureau fédéral de la Statistique, «Caractéristiques de la population et du logement par secteur de recensement - Montréal», Recensement du Canada, 195I, Bulletin CT-3 (juin 1953), 2442; Bureau fédéral de la Statistique, «Caractéristiques de la population et du logement par secteur de recensement - Montréal », Recensement du Canada, 1961, Catalogue 95-519, Bulletin CT-4 (août 1963), 30-5I ; Statistique Canada, «Caractéristiques de la population et du logement par secteur de recensement - Montréal », Recensement du Canada, 197I, Catalogue 95-734, Bulletin CT-4B (octobre 1974), 126-169. Les données sur les revenus contenues dans les publications de Statistique Canada ne nous permettent pas de comparer un même endroit dans le temps. À noter que l'idée de comparer les revenus de la Cité de Montréal et des banlieues vient de Séguin et al. qui ont toutefois opté, au lieu d'un tableau, pour une représentation cartographique où ils illustrent le gain médian des chefs de famille salariés en 195 I et le revenu médian des ménages en 1971. Séguin et al., «Pauvreté et richesse dans la région montréalaise depuis l'après-guerre... ॥, I| 47 - | | 53. 
Cet étalement de la pauvreté n'empêche pas la précarité matérielle de s'incarner plus particulièrement dans certaines parties de la ville au tournant des années 1950. En 1951, les chefs de famille de la majorité des banlieues de l'île ${ }^{26}$ affichent déjà un revenu médian nettement supérieur à celui des habitants de la Cité de Montréal (2374\$). L'écart est particulièrement marqué dans le cas des banlieues regroupant traditionnellement les Canadiens anglais et situées dans l'ouest de la ville, notamment CôteSaint-Luc (où le revenu médian est de $3765 \$$ ), Hampstead (5926\$), Montréal-Ouest (5083\$), Mont-Royal (5747\$) et Westmount (3445\$) Dix ans plus tard, les revenus des chefs de famille salariés montrent que la tendance se maintient: si, dans la Cité de Montréal, il tourne autour de 3962 \$, il atteint 4407 \$ dans la zone métropolitaine de Montréal, et se situe entre 7119 \$ et 10311 \$ dans les banlieues de l'île énumérées plus haut ${ }^{28}$. En 1971, l'écart s'amplifie entre les chefs de famille de la Cité de Montréal et ceux de la majorité des municipalités adjacentes, c'est-à-dire, entre les chefs de famille qui demeurent sur le territoire montréalais et ceux qui ont les moyens financiers de migrer vers les banlieues. Non seulement les clivages entre les banlieues anglophones plus aisées (où le revenu moyen de l'emploi du chef de famille salarié oscille entre 12122 \$ et 19778 \$) et le cour de la ville (ce même revenu est de 6796 ) perdurent, voire s'accentuent, mais comparativement à la situation qui prévaut en 1961, le fossé se creuse entre les revenus de la Cité de Montréal et ceux de municipalités comme Outremont (11 209\$), Saint-Laurent (9077\$), SaintLéonard $(7658$ \$) et Pierrefonds (10 062 \$).

L'analyse des données des recensements sur la distribution des revenus en 1951 et en 1971 par Séguin et al. corrobore cet accroissement du fossé "entre un centre pauvre et une banlieue mieux nantie ${ }^{29}$ ». En effet, la division en quintiles du revenu médian des ménages salariés permet de constater qu'au début des années 1970, la quasi-totalité des «secteurs à faible revenu sont concentrés dans le centre [et] correspondent grosso

26. Nous avons retenu les municipalités ayant une frontière avec la Cité de Montréal, à l'exception de Saint-Jean-de-Dieu, une sorte de cité-hôpital qui doit être considérée à part.

27. Selon le recensement de 1951 , la proportion de la population (se disant) d'origine britannique est de $56,42 \%$ à Côte-Saint-Luc, de 60,06\% à Hampstead, de 82,05\% à Montréal-Ouest, de $64,76 \%$ à Mont-Royal et de $63,47 \%$ à Westmount. Elle est de $22,02 \%$ et de $17,67 \%$ pour, respectivement, la zone métropolitaine de Montréal et la Cité de Montréal proprement dite. Bureau fédéral de la Statistique, "Caractéristiques de la population et du logement par secteur de recensement - Montréal ", Recensement du Canada, 1951, Bulletin CT-3 (juin 1953), 4-17.

28. Les données ne nous permettent pas de spécifier s'il s'agit du revenu moyen ou médian.

29. A.-M. Séguin et al., "Pauvreté et richesse dans la région montréalaise depuis l'après-guerre... », loc. cit., 1151. 
modo aux quartiers Saint-Henri, Pointe-Saint-Charles, à la portion sud du centre-ville (la rue Sherbrooke correspondant à sa limite nord), aux quartiers Hochelaga-Maisonneuve, Centre-Sud, Plateau Mont-Royal, Mile End, Parc-Extension et La Petite Patrie ${ }^{30}$ ». Les revenus des ménages sont aussi sous la médiane dans les quartiers Côte-des-Neiges et le sud de NotreDame-de-Grâce (tous deux dans le secteur centre-ouest de la Cité de Montréal).

\section{QUI SONT CES EXCLUS DE LA PROSPÉRITÉ EN MILIEU URBAIN?}

If you have the misfortune to be a woman, an Indian, an Eskimo or a FrenchCanadian, your chances of being poor are greater-much greater - than if you were young, white, male and English speaking .

Ces inégalités qui se traduisent par la précarité matérielle de ceux et celles qui en font les frais ne sont évidemment pas que géographiques: souvent dans ces quartiers, parfois à l'extérieur de ceux-ci, habitent des individus dont l'identité attire l'attention des contemporains des Trente Glorieuses. Entre 1967 et 1970, la Commission royale d'enquête sur le bilinguisme et le biculturalisme (Laurendeau-Dunton) mise sur pied en 1963 publie son rapport où elle aborde entre autres la question «des aspects socio-économiques de l'égalité ${ }^{32}$ » entre les francophones et les anglophones au pays. Elle révèle à quel point les premiers sont désavantagés par rapport aux seconds, qu'il s'agisse des revenus, du niveau d'instruction, de leur présence respective aux échelons supérieurs des secteurs privés ou publics, du contrôle de l'économie ou de la langue de travail. En effet, à l'échelle du pays, les hommes canadiens-français gagnent environ $80 \%$ du revenu de leurs homologues d’origine britannique au début des années 1960, cette disparité étant nettement plus prononcée au Québec ${ }^{33}$.

Si l'infériorité économique des Canadiens français pendant au moins une partie des Trente Glorieuses est indéniable, ils ne sont pas les seuls dans cette situation, l'historiographie ayant noté la persistance pendant l'après-guerre «de profondes inégalités entre les groupes ethniques et

30. Ibid.

31. Ian Adams et al., The Real Poverty Report (Edmonton, M. G. Hurtig Limited, 1971), 68.

32. Commission royale d'enquête sur le bilinguisme et le biculturalisme, Rapport (Laurendeau-Dunton) de la Commission royale d'enquête sur le bilinguisme et le biculturalisme, Livre III : Le monde du travail (Ottawa, Imprimeur de la Reine, 1969), 3.

33. Ibid., 18-24 et Livre IV : L'apport culturel des autres groupes ethniques, p. 599 (Ottawa, Imprimeur de la Reine, 1969). 
sociaux, entre les sexes et entre les régions ${ }^{34}$ ». Paul-André Linteau, René Durocher, Jean-Claude Robert et François Ricard soulignent ainsi que les Canadiens d'origine italienne partagent le sort des Canadiens français en tant que «citoyens de seconde zone ${ }^{35}$ » dont les revenus sont moins élevés que la moyenne. Jocelyn Létourneau abonde un peu dans le même sens: bien qu'il rapporte que, selon le recensement de 1961, les hommes salariés issus des diverses communautés culturelles sont, côté salaire, dans l'ensemble en meilleure posture ${ }^{36}$ que leurs concitoyens «de souche francophone», il remarque le désavantage économique des Italiens et des Autochtones $^{37}$. En font foi les données de la Commission LaurendeauDunton qui illustrent qu'au Québec, les salariés masculins des Premières Nations gagnent en moyenne 2112 \$ par année, comparativement à 2938 \$ pour les Canadiens italiens, $3185 \$$ pour les Canadiens français et $4940 \$$ pour les Canadiens anglais. Toutes origines confondues, la moyenne des gains des hommes s'élève alors à $3469 \$$ dans la province ${ }^{38}$.

Cette inscription dans l'espace de l'infériorité des revenus de certains groupes ethniques, en premier lieu des Québécois francophones, imprègne aussi le territoire montréalais. Plus précis que nos données nous permettent de l'être, Martial Fauteux, dans un rapport produit pour l'Office de planification et de développement du Québec à la fin des années 1970, remarque que les familles dites «à faible revenu » se concentrent le long des lignes de faille ethniques en « une zone francophone pauvre le long du fleuve dans le nord-est, une zone d'immigrants le long du boulevard Saint-Laurent dans le nord-ouest, et enfin une zone qui suit le canal Lachine et dont la population est principalement composée de francophones et d'Irlandais catholiques romains ${ }^{39}$ ». Plus encore, comme l'indique en 2012 Mario Polèse, les écarts de revenus entre francophones et anglophones, dont les données du recensement de 1961 permettent pour la première fois de prendre vérita-

34. P.-A. Linteau et al., Histoire du Québec..., op. cit., 205.

35. Ibid.

36. Plus précisément, en s'appuyant sur les données censitaires rapportées par la Commission Castonguay-Nepveu, Létourneau souligne que «le revenu de travail (qui est la composante la plus importante du revenu global) des salariés masculins d'origine ethnique française s'élève à $64.5 \%$ seulement du revenu de travail des salariés masculins d'origine britannique [et] à $91.8 \%$ du revenu de travail des salariés de toutes origines ethniques dans la province», Jocelyn Létourneau, Accumulation, régulation et sécurité du revenu au Québec au début des années 1960, thèse de doctorat (histoire), Université Laval, 1984, 261.

37. Ibid., 256-263.

38. André Raynaud, Gérald Marion et Richard Béland, La répartition des revenus selon les groupes ethniques au Canada. Tableau reproduit dans Commission royale d'enquête sur le bilinguisme et le biculturalisme, Rapport (Laurendeau-Dunton) de la Commission..., Livre III : Le monde du travail, op. cit., 23.

39. Martial Fauteux, Les revenus (Québec, Office de planification et de développement du Québec, 1979), 27. 
blement la mesure, révèlent qu’ «[à] Montréal, la rémunération moyenne d'un travailleur francophone unilingue équivaut à la moitié de celle d'un unilingue anglophone ${ }^{40}$ ». Au fil du temps et des politiques sociales, notamment dans la foulée de la Révolution tranquille qui hausse le niveau d'éducation de bien des Franco-Québécois, cet écart s'amenuise considérablement, mais perdure au-delà de 1975 .

La communauté noire de Montréal n’est pas épargnée non plus. Groupée près de la rue Saint-Antoine dans le quartier Saint-Henri situé dans le sud-ouest de la ville, elle fait l'objet de discrimination en matière d'emploi et de logement, comme le souligne Sean Mills dans son portrait de la ville dans les années $1960^{41}$. Ses membres jouissent en moyenne d'un an d'éducation de plus et d'un salaire (à peine) plus élevé que les Canadiens français en 1961, ce qui ne suffit évidemment pas pour leur faire oublier les pratiques discriminatoires et le racisme dont ils sont l'objet ${ }^{42}$.

Cela dit, comme l'indique le journaliste et écrivain canadien-anglais Ian Adams en 1970, «[t]here is no doubt that the poorest of the poor in this country are the Indians, the Eskimos, and the Metis ${ }^{43}$ ». En ce qui concerne Montréal, il ne fait aucun doute non plus que des individus ayant des origines autochtones ou se définissant comme tels habitent la ville pendant les Trente Glorieuses même si les sources nous permettent très rarement de les identifier. Certes, au début des années 1950, les Autochtones se comptent par centaines dans la plupart des centres urbains du pays, mais une certaine urbanisation des individus vivant auparavant sur des "réserves» ou des territoires autochtones a lieu au cours de la décennie suivante ${ }^{44}$. Si le phénomène est particulièrement marqué dans l'Ouest canadien, ce sont tout de même 3215 Montréalais que le recensement de 1971 identifie comme "Indiens nord-américains ${ }^{45}$ ». De surcroit, le rapport de Martial Fauteux note l'existence de zones de pauvreté en périphérie de Montréal, notamment à

40. Mario Polèse, "Montréal économique: de 1930 à nos jours. Récit d'une transition inachevée», dans Dany Fougères, dir., Histoire de Montréal et de sa région, Tome II : De 1930 à nos jours (Québec, Les Presses de l’Université Laval, 2012), 976.

41. Sean Mills, The Empire Within. Postcolonial Thought and Political Activism in Sixties Montreal, (Montréal et Kingston, McGill-Queen’s University Press, 2010), 36, 95.

42. David Austin, Fear of a Black Nation. Race, Sex, and Security in Sixties Montreal (Toronto, Between the Lines, 2013), 51.

43. Ian Adams, The Poverty Wall (Toronto, McClelland and Stewart, 1970), 27.

44. Mary Jane Norris et Stewart Clatworthy, «Urbanization and Migration Patterns of Aboriginal Populations in Canada. A Half Century in Review (1951 to 2006)", Aboriginal Policy Studies, 1, 1 (2011): 30-31.

45. Statistique Canada, «Population-Groupes ethniques», Recensement du Canada, 1971, Catalogue 92-723, Vol. 1 partie 3, Bulletin 1.3-2 (octobre 1973). 
Kahnawà :ke $\mathrm{k}^{46}$. Les quelques données dont nous disposons sur les Autochtones corroborent la situation que laissent entrevoir les chiffres de la Commission Laurendeau-Dunton mentionnés plus haut. L'étude du revenu annuel moyen, et non seulement des salaires, révèle un portrait encore plus sombre: en 1963, il s'élève à 1600 \$ pour les membres des Premières Nations contre $3500 \$$ dans l'ensemble du pays ${ }^{47}$. Le très controversé Livre blanc sur la politique indienne du gouvernement fédéral réitère, en 1969, la pauvreté qui afflige une proportion plus grande d'Autochtones que de non-Autochtones ${ }^{48}$. Ainsi, le militant et homme politique autochtone Walter Deiter a très probablement raison d'affirmer au tournant des années 1970 que «les autochtones [sic] ne sont pas tous pauvres, mais l'on peut désigner ceux qui le sont comme "les plus pauvres d'entre les pauvres"49".

La pauvreté touche aussi durement les femmes, sans doute plus nombreuses que les hommes dans les rangs des défavorisés au Canada, comme le note en 1970 le rapport de la Commission royale d'enquête (Bird) sur la situation de la femme; celui-ci révèle qu’en 1967, «[p]lus d'un tiers de toutes les femmes chefs de famille sont des économiquement faibles ${ }^{50}$ ». Qui plus est, ces ménages dirigés par des femmes représentent 7,5\% des familles, mais comptent pour «14.8 [sic] pour cent des familles à faible revenu». Ce n'est guère surprenant quand on tient compte des écarts entre le revenu des hommes et des femmes chefs de famille: en 1967, à l'échelle du pays, les premiers gagnent en moyenne $5821 \$$, comparativement aux $2536 \$$ dont doivent se contenter les secondes (et leurs dépendants) ${ }^{51}$. Nous ne disposons pas de données sur les écarts de revenus entre les femmes et les hommes chefs de famille avant cette date. Le Collectif Clio fait toutefois ressortir la persistance d'un écart analogue entre les travailleuses et les travailleurs en général au début du $\mathrm{XX}^{\mathrm{e}}$ siècle alors que «les femmes touchent en moyenne la moitié des salaires masculins [: à] Montréal, elles reçoivent 53,6 p. 100 du salaire des hommes en 1921, 56,1 p. 100 en 1931

46. M. Fauteux, Les revenus..., op. cit., 27.

47. W. Rudnicki, «The Big Picture. Indian Affairs Branch Statement for Federal-Provincial Conference on Poverty, November 1965 ", cité dans Sally M. Weaver, Making Canadian Indian Policy. The Hidden Agenda, 1968-1970 (Toronto, University of Toronto Press, 1981), 26 cité dans Jacques Paul Couturier en collaboration avec Wendy Johnson et Réjean Ouellette, Un passé composé. Le Canada de 1950 à nos jours (Moncton, Éditions d'Acadie, 2000 [1996]) 318.

48. Ministère des Affaires indiennes et du Nord canadien du Canada, La politique indienne du gouvernement du Canada, 1969 (Ottawa, Imprimeur de la Reine, 1969).

49. D. A. Croll, La pauvreté au Canada..., op. cit., 37.

50. Commission royale d'enquête sur la situation de la femme au Canada, Rapport de la Commission royale d'enquête (Bird) sur la situation de la femme au Canada (Ottawa, Information Canada, 1970), 361.

51. Bureau fédéral de la statistique, Enquête sur la situation matérielle du consommateur, 1968 cité dans ibid., 348 et 361 . 
et 51 p. 100 en $1941^{52}$ ». Rien n'indique que le patriarcat s'incarne moins dans la rémunération de la main-d'œuvre dans les années 1940 et 1950. Bien au contraire, si l'on tient compte des tentatives des autorités de renvoyer les travailleuses de guerre au foyer une fois la guerre terminée et, de manière plus générale, du caractère contesté du droit au travail salarié des femmes jusqu'aux années 1960 au moins ${ }^{53}$. Cette disparité dans les salaires, conjointement aux inégalités qui défavorisent les Autochtones et les autres communautés ethniques, fait donc en sorte "que les plus dénuées sont les Indiennes, les Métisses et les Esquimaudes $[s i c]^{54}$ », suivies par les Noires, les Canadiennes italiennes et les Canadiennes françaises.

Qu'on soit une femme, un homme, un Autochtone, un Canadien français ou anglais, détenir un emploi rémunéré ne suffit pas pour entrer dans la consommation de masse pendant les Trente Glorieuses. Comme le souligne le rapport de la Commission Bird, «les données du Bureau fédéral de la Statistique pour 1967 indiquent que deux tiers des chefs de famille économiquement faibles [font] partie de la population active pendant au moins une partie de l'année, et qu'un tiers d'entre eux [ont] travaillé à temps plein toute l'anné $e^{55}$ ", ce qui en dit long sur l'insuffisance des salaires. Pire encore, l'universitaire Émile Gosselin, dans La troisième solitude, affirme que «[s]eize et demi $\left(16 \frac{1}{2} \%\right)$ pour cent des chefs de famille salariées du Canada et dixsept et demi $\left(17 \frac{1}{2} \%\right)$ pour cent des chefs de famille salariées du Québec ne jouissent pas de conditions de vie suffisantes pour leur permettre de satisfaire même leurs besoins essentiels ${ }^{56}$ " au début des années 1960. En ce qui concerne la zone métropolitaine de Montréal, le revenu de 11,3\% des chefs de famille salariés se trouve sous le seuil de pauvretée ${ }^{7}$.

Un dernier mot s'impose sur le rôle joué par la taille de la famille dans la situation financière d'un ménage. Comme l'indique Gosselin, «les

52. Le Collectif Clio, L’histoire des femmes au Québec depuis quatre siècles (Montréal, Les Quinze, 1982), 270. Sur la persistance de la discrimination salariale défavorisant les femmes, voir aussi: Denyse Baillargeon, Brève histoire des femmes au Québec (Montréal, Boréal, 2012), 68-70, 97, 185 et 222-223.

53. Le Collectif Clio, L'histoire des femmes au Québec..., op. cit., 205-207, 270-271, 384, 443; Geneviève Auger et Raymonde Lamothe, De la poêle à frire à la ligne de feu. La vie quotidienne des Québécoises pendant la guerre 39-45 (Montréal, Boréal Express, 1981) 160 ; D. Baillargeon, Brève histoire des femmes, op. cit., 156157; Renée Dandurand, Le mariage en question. Essai socio-historique (Québec, IQRC, 1991 [1988]), 26; Ruth Roach Pierson, "They're Still Women after All». The Second World War and Canadian Womanhood (Toronto, McClelland and Stewart, 1986).

54. Commission royale d'enquête sur la situation de la femme au Canada, Rapport de la Commission, op. cit., 371.

55. Ibid., 351.

56. E. Gosselin, La troisième solitude..., op. cit., 17.

57. Ibid. 
familles sans dépendants ou avec 4 dépendants ou plus ${ }^{58}$ » ont plus de chances d'être pauvres. Le rapport du Comité spécial du Sénat sur la pauvreté conforte cette assertion, dans la mesure où les familles canadiennes comprenant 6,2 membres (c'est-à-dire la taille moyenne des unités familiales comprenant 5 personnes ou plus) affichent un taux de pauvreté de $28,5 \%$ (par rapport à un seuil de pauvreté à 6570 \$) à la fin des années 1960. Ce taux équivaut presque au double de celui des familles de 3 et de 4 personnes (dont le seuil de pauvreté s'établit respectivement à 4290 \$ et $5000 \$$ ) qui oscille autour de $15-16 \%$. En ce qui concerne les familles «très nombreuses » de sept ou huit personnes, cette proportion «est de près de 40 à 50 p. cent $»^{59}$. Même si le travail salarié d'un plus grand nombre d'enfants peut contribuer à améliorer les finances familiales, il est bien évident qu'un chef de famille qui a huit, voire neuf ou dix bouches à nourrir se trouve en moins bonne posture que celui qui, gagnant un montant équivalent, en a six ${ }^{60}$.

En fin de compte, ce bref survol de la pauvreté en temps de prospérité montre bien la persistance de ce phénomène à Montréal pendant la période d'abondance relative qui s'étire de 1945 à 1975. Certes, la période ne peut être construite comme uniforme. La rareté des sources sur les ménages identifiés comme pauvres pendant les années 1940 et 1950, probablement parce que leurs conditions de vie apparaissent moins décalées par rapport à celles de la majorité, rend ardue une analyse longitudinale. Il reste que pendant une périodisation dont la cohérence réside dans la prospérité économique, une pauvreté colore le quotidien de ménages montréalais, même si elle le teinte de manière très certainement différente en 1945 qu'en 1975. La plus grande prospérité et l'accès des masses à la société de consommation altèrent sans doute l'expérience des ménages qui s'en trouvent laissés pour compte. Un recours aux témoignages serait

58. Ibid., 51.

59. D. A. Croll, La pauvreté au Canada..., op. cit., 13-15.

60. La taille moyenne des ménages québécois varie entre 3,7 et 4,6 personnes entre 1951 et 1971. En ce qui concerne la zone métropolitaine de Montréal, les ménages sont un peu plus petits: ils comptent en moyenne 4 individus en 1951, 3,7 en 1961 et 3,3 en 1971. Bureau fédéral de la Statistique, «Caractéristiques de la population et du logement par secteur de recensement - Montréal», Recensement du Canada, 1951, Bulletin CT-3 (juin 1953), 24; Bureau fédéral de la Statistique, "Caractéristiques de la population et du logement par secteur de recensement - Montréal», Recensement du Canada, 1961, Catalogue 95-519, Bulletin CT-4 (août 1963), 30; Bureau fédéral de la Statistique, «Revue générale. Taille et composition du ménage », Recensement du Canada, 1961, Catalogue 99-529, Bulletin 7.2-3 (novembre 1967), 8-5; Statistique Canada, "Caractéristiques de la population et du logement par secteur de recensement - Montréal», Recensement du Canada, 1971, Catalogue 95-704, Bulletin CT-4A (mai 1973), p. 4; Statistique Canada, «Ménages selon la taille», Recensement du Canada, 1971, Catalogue 93-702, Bulletin 2.1-2 (mai 1973), 1-1. 
nécessaire pour bien prendre le pouls de cette transformation de la pauvreté pendant les Trente Glorieuses.

Malgré ces lacunes, quelques conclusions peuvent être tirées. Le taux de pauvreté, bien qu'il constitue une construction imparfaite - comme toute forme de donnée statistique, d'ailleurs - laisse entrevoir que l'insuffisance du revenu touche plus ou moins, selon le moment, entre 11 et $20 \%$ des ménages de la ville. Plus encore, ces écarts épousent les divisions de la société montréalaise, les clivages linguistiques et ethniques s'incarnant sans grande surprise dans la géographie de la pauvreté. Les Canadiens français, les Canadiens italiens tout comme les membres de la communauté noire se trouvent ainsi particulièrement désavantagés tout comme les Autochtones dont nous savons bien peu des conditions de vie à Montréal après la Seconde Guerre mondiale. Autre constat: les ménages ayant pour chef une femme ainsi que les familles nombreuses ont eux aussi plus de chances de connaître des difficultés économiques. Outre le fait que bien des pauvres appartiennent aux rangs des salariés, ces résultats n'étonnent guère aujourd'hui, ce qui reflète l'aspect tristement «normal» que cette pauvreté - dont les caractéristiques que nous avons mises en lumière perdurent, hormis dans le cas des Franco-Québécois - revêt en ce début de $\mathrm{XXI}^{\mathrm{e}}$ siècle. Il faut toutefois garder en tête que pendant les Trente Glorieuses, surtout à partir des années 1960, l'exclusion d'une frange de la population du giron de la société de consommation n'a rien d'inévitable ou de normal. Au contraire, surtout dans les années 1960 et 1970, cette injustice est perçue comme problématique ${ }^{61}$. 